\title{
Review of: "MLF2 modulates phase separated nuclear envelope condensates that provoke dual proteotoxicity"
}

\author{
Jihane Basbous ${ }^{1}$ \\ 1 French National Centre for Scientific Research
}

Potential competing interests: The author(s) declared that no potential competing interests exist.

The article "MLF2 modulates phase-separated nuclear envelope condensates that cause dual proteotoxicity" explores the contribution of NE blebs in the manifestation of DYT1 dystonia and describes two proteotoxic mechanisms that could explain the etiology of the disease. Several cellular and biochemical approaches were used to support their hypothesis which is very interesting. The article is well constructed even if several paragraphs can be merged. On the other hand, some experimental approaches are problematic and there is a lack of scientific rigor necessary to convince the reader. Below are my comments and questions that I hope will help authors improve the quality of their manuscript.

\section{Figure 1:}

The scale bars of several Immunofluorescence panels are not added.

- 1b: There is a duplicate of figure $1 \mathrm{~b}$ and $1 \mathrm{~d}$ of the western blot (with anti-HA antibody) with a slightly different legend.

- 1d: The authors clearly demonstrated that the D133-ORF10-HA protein is more stable in TorsinKO than WT cells. How do the authors explain that the immunoprecipitation of the protein in 2 cellular contexts (figure 1d, last panel) is at the same level?

- 1f: The statistics are missing.

- 19: Does the expression level of the two constructs D133 ORF10- M48-HA and D133 ORF10- M48-HAC23A-HA vary. It will be better if the authors add the western blot panel.

\section{Figure 2:}

- 2c: Why the blebs in TorsinKO cells (the 5 cells chosen by the authors) are not so obvious compared to the immunofluorescence of figure 1 ( $c$ and $g$ ) or other IF panels.

- 2e and f: I have a question concerning the Mass spec of the IP D133-ORF10-HA. Is there a reason why DNAJB6 was not found in the mass spec of the IP when you are observing its interaction by western blot? 
Do you think that the results may be biased by the fact that the proteins (IP D 133-ORF10-HA or even MLF2 see western blot $2 \mathrm{~b}$ ) are more stable in TorsinKO background?

\section{Figure Extended data 1:}

- 1a: The authors state that there is little recruitment of DNAJB12-HA to the blebs. It is difficult to confirm this on a single cell basis. The authors should represent a line scan to confirm whether or not the 2 proteins colocalize or the Pearson correlation coefficient to quantify the degree of colocalization between the two proteins.

\section{Figure 3:}

- 3a: In the same line, the authors claim that HSPAA, HSC70, DNAJB6 and DNAJB2 proteins localize to blebs without showing any colocalization with the K48-Ub. The data are not so convincing. Why DNAJB6 shows a scattered distribution?

- 3c: The arrowheads are missing in TorsinKO Hela.

\section{Figure 5:}

It's better to show the efficiency of MLF2 depletion by western blot

- 5a: Why the authors do not show the colocalization between K48- Ub and HSPA1A or DNAJB6 in MLF2 knockdown cells? It will be more informative to see that DNAJB6 is no more present to blebs stained by K48-Ub.

- 5b: I didn't get the relevance of this experiment. The authors speculate the name of the proteins following their molecular weight but there is no direct evidence. Lane 142: They made conclusions very quickly without solid evidence. One control is missing in this model: the depletion of MLF2 in TorsinWT cells.

- 5c: The immunoprecipitation of this Figure is sufficient and more conclusive than the assay of figure $5 \mathrm{~b}$. It's better to show the MLF2 input and whether MLF2 immunprecipitates with HSPA1A (confirming the mass spec Data of figure 2e) and again the depletion of MLF2 in TorsinWT is missing.

Another observation, does the depletion of MLF2 by means of siRNA destabilizes DNAJB6? In other term, the input of the figure tends towards this hypothesis. If this is the case, the depletion of MLF2 in the TorsinWT is important to do. Depending on the result, the conclusion must be corrected. Indeed, if MLF2 depletion affects the stability of the DNAJB6 protein, this may exclude its role in the localization of DNAJB6 into the blebs

- 5e: The authors state that in TorsinKO/MLF2 KO cells, DNAJB6 is recruited to sites of poly-Q aggregation and after reintroducing MLF2-HA, DNAJ6 is sequestered into blebs. However, when we observe the right middle panel of figure5e, DNAJ6 is distributed throughout the nucleoplasm and not into blebs. Is there an 
explanation for this observation?

\section{Figure 6:}

- 6c: The authors show the efficiency of siRNA depletion by qPCR, however, it is preferable to show the protein level of endogenous NUP98 after siRNA transfection and also to see if NUP98 smartPool oligos affect the level of recombinant HA-NUP98 protein. In Extended data 2C, the expression of recombinant HA-NUP98 shows, in addition to a localization in the nucleoplasm, a nucleolar localization in siControl transfected cells but this nucleolar localization disappears completely after siNUP8 transfection with a decrease in nucleoplasm intensity.

NUP96 shows a cytosolic staining, is it the best control?

- Extended data Fig 2e: The authors conclude that HSPAIA and HSC70 do not localize to the cytosolic puncta. However, their expression in less intense than GFP-MLF2 or DNAJB6, it's interesting to do a line scan or a colocalization with a recombinant HSPA1A or HSC70 to be sure that that they do not colocalize to the cytosolic puncta.

- 6f: In the phase separation field, great care should be taken when using Hexanediol in living cells. It is very controversial to make a conclusion on condensates formed by liquid liquid phase separation process based only on experiments with Hexanediol in living cells.

DOI: $10.1016 /$ j.jbc.2021.100260

DOI: $10.26508 /$ lsa.202001005

\section{Figure 7}

- 7b : It's important to show whether the expression of MLF2 mutants (that lacks arginine or methionine) is the same than MLF2 WT. 